\title{
Appendix 3: The German Draft of the Philosophie Sociale
}

The transcription is based on the autograph preserved in Paris, Archives Nationales, $T 1524$ (for a brief description of the entire set of papers, confiscated from Dobruska upon arrest, see Susanne Wölfle-Fischer, Junius Frey (1753-1794). Jude, Aristokrat und Revolutionär, (Frankfurt a. M.: Peter Lanf, 1997), 20-26.

\begin{tabular}{ll}
\hline CHAPITRE VIII. & Abtheilung VIII \\
\hline Du souverain. & Der Souverain \\
\hline I. & I. \\
\hline $\begin{array}{l}\text { Qu'est-ce que l'être-homme dans l'état de } \\
\text { nature? Un ensemble souverain, un tout } \\
\begin{array}{l}\text { parfait et isolé dépendant de soi-même et } \\
\text { indépendant hors de soi. }\end{array}\end{array}$ & $\begin{array}{l}\text { Was ist d[er] [.] Naturmensch? - ein einzelnes } \\
\text { independentes Souveraines Ganze (C. §) }\end{array}$ \\
\hline
\end{tabular}

II.

2.

Qu'est-ce que c'est que la société ? Un nouvel ensemble ou un tout souverain et

Was ist die Sozietät? - ein vo[n] einzelnen, independenten, in Rechten gleichen indépendant, spontanément composé

d'ensembles primitifs isolés, aussi Souverainen ältern Ganzen, freiwillig indépendans souverains et égaux en droits, multipliés d'une manière égale et homogène, zusammengestelltes neues Ganze, gleichartig vervielfältigt aber nicht verschiedentlich vermehrt.

mais non pas augmentés différemment.

III.

3.

Que reçoit le souverain primitif (l'être homme) dans l'état de nature du nouveau souverain, ( la société) il en reçoit la faculté appétitive tant matérielle qu'intellectuelle perfectionnée et développée.

Was erhält der alte Souverain (: der individuelle Naturmensch) von dem neuen Souverain (: die Sozietät)? - das erhöhte und entwickelte materielle und intellektuelle Begehrungsvermögen (C. I.§4. C.II.§.4).

IV.

4.

Que doit-on entendre par la faculté appétitive, matérielle et intellectuelle perfectionnée et développée? L'instinct mûri et formé par la société.

Was ist der erhöhte und entwickelte materielle und intellektuelle Begehrungsvermögen? - es ist der großgewachsene von der Gesellschaft erzogene, ausgebildete Instinkt. 
(continued)

\section{CHAPITRE VIII.}

V.

Quelle est la propriété de l'être-homme dans l'état de nature? toutes les forces et moyens naturels qui peuvent le conduire au but exigé par le principe; c'est-à-dire, au plus parfait contentement de sa faculté appétitive animale et non développée, qui n'est autre chose que l'instinct, par lequel dans cet état, le principe dirige toutes ces forces et moyens naturels qui combinés ensemble, constituent la propriété du souverain primitif.

VI.

En quoi consiste la propriété du nouveau souverain? (la société) dans toutes les forces et moyens de culture, qui se réunissent aux forces et moyens naturels antérieurs de chacun de ses membres, pour étendre la sphère de ses jouissances par le plus parfait contentement de sa faculté appétitive tant matérielle qu'intellectuelle perfectionnée et développée; et c'est la réunion de ces forces et moyens de culture qui constitue la propriété du nouveau souverain. La société

VII.

Quels sont les devoirs et fonctions du souverain primitif (l'être-homme dans l'état de nature) en vers lui-même ? La contribution et l'emploi de toutes ses forces et moyen naturels pour atteindre le but qu'exige le principe, c'est-à-dire le plus parfait contentement de la faculté appétitive non développée, et de l'instinct, qui réunis, constituent la propriété du souverain primitif, et qui doivent s'accorder une protection réciproque et s'entre-aider mutuellement.

VIII.

Jusqu'où s'étendent les devoirs et obligations du souverain primitif; (c'est-à-dire l'êtrehomme dans l'état de nature) en vers

\section{Abtheilung VIII}

\section{5}

Was ist das Eigenthum des alten Souverains? (des individuellen Naturmenschen)? - alle seine Kräfte und Naturmittel zu Erreichung seines eingeschränkten Zwecks - die höchsten Befriedigung seiner unentwickelten animalischen Begehrungsvermögen welches der Instinkt ist, <so> der Leiter dieser Kräfte und Naturmittel ist, und mit ihren zusammen das Eigenthum des alten Souverains ausmacht.

\section{6.}

Was ist das Eigenthum des neuen Souverains (die Sozietät)? - alle <zu seinen alten Kräften und Naturmitteln hinzugekommen> neue Kräfte und Kulturmittel zu Erreichung des ausgedehnteren Zweckes - das höchsten Befriedigung ihres höchsten und entwickelten materiellen und intellektuellen

Begehrungsvermögen (84) welches der Leiter dieser neuen entwickelten Kräfte und Kulturmittel ist, und mit ihren zusammen das Eigenthum des neuen Souverains (die lieson soziale \{liaison sociale\}) ausmachen.

\section{7.}

Was sind die Pflichten des alten Souveraines (das individuelle Naturmenschen) gegen sich selbst? - sie sind die Beisteuer aller seiner Kräfte und Naturmittel zu Errichtung ihres eingeschränkten Zweckes - der höchsten Befriedigung ihres animalischen Leiters, des Instinktes, welcher mit ihren zusammen das Eigenthum des alten Souverains (95.) ausmacht, die sich untereinander Hülfe und reziproken Schutz leisten müßen.

8.

Wie weit gehen die Pflichten des alten Souverains (des individuellen Naturmenschen) gegen sich selbst? - So weit, 
(continued)

\begin{tabular}{|c|c|}
\hline CHAPITRE VIII. & Abtheilung VIII \\
\hline $\begin{array}{l}\text { soi-même? Ils s'étendent autant que ses } \\
\text { moyens, c'est-à-dire autant que restant } \\
\text { moyens ils ne touchent point au but. D’où il } \\
\text { résulte que toutes ses prétentions qui } \\
\text { touchent au but sortent du cercle de son juste } \\
\text { pouvoir et dégénèrent en usurpation } \\
\text { tyranniqe, parce que c'est alors contre le } \\
\text { principe; tels que le suicide qu'il ne doit point } \\
\text { commettre, quoiqu'il soit en son pouvoir de } \\
\text { de le faire, }\end{array}$ & $\begin{array}{l}\text { als sie Mittel bleiben, und nicht mit dem } \\
\text { Zweck vertauscht werden; daher alle } \\
\text { Forderungen sobald sie den Zweck berühren, } \\
\text { außer der Souverains Gerechtigkeit liegen, } \\
\text { und werden zur Usurpazion und Tirannie, zum } \\
\text { Selbstmord, welchen - ob er ihn gleich } \\
\text { ausüben kann - doch sicher nicht ausüben } \\
\text { darf. }\end{array}$ \\
\hline
\end{tabular}

IX.

9.

Quelle est la conduite du souverain primitif dans cette contribution de ses forces et moyens naturels? je veux dire les employe-t-il avec une économie scrupuleuse ou avec profusion? Économise-t-il cette contribution, quand il s'agit de défendre sa propriété pour ne pas manquer à son but exigé par le principe? Non. Il suit constamment la loi invariable qui dit, que cela soit. La victoire ou la mort; et il s'acquitte involontairement de sa contribution, tant qu'il lui reste un moyen de défendre cette propriété relativement au but exigé par le principe, car tous ses moyens tendans vers ce but, n'ont qu'une existence relative à lui et finissent aussitôt qu'il cesse d'exister; d'ailleurs, l'instinct ou élan tendant à obtenir ce but, est tellement lié à l'instinct conservateur de leur propre existence, au principe, qu'ils sont obligés de déployer toutes leurs ressources pour ne pas périr euxmêmes en perdant leur but: je veux dire pour ne pas se perdre absolument, ayant encore l'espoir de parvenir à conserver leur existence, par la possibilité de triompher et d'atteindre le but.
Wie benimmt sich der alte Souverain (der individuelle Naturmensch) in dieser Beisteuer seiner Kräfte und Naturmittel, d.i. giebt er sie kärglich, oder genugsam, geitzt und [.] in Ansehung dieser Neuer, wenn es daraus ankommt \{ankommt\} sein Eigenthum zu stützen, um seines Zweckes nicht verlustig zu werden? - nein! Er weichet nicht in geringsten $a b$, von seinen unerschütterlichen Gesetzen, das da heißt: so und nicht anders! Ding, ohne Vermischung! - er leistet seinen Steuer (die Mittel) unwillkürlich, und zwar in so lange, was ihm nur noch ein einziges Mittel übrig bleibt, dieses Eigenthum, um des Zweckes willen zu beschützen; denn da alle seine Mittel zu Erreichung dieses Zwecks nur eine für diesen Zweck relative Existenz haben, und sogleich aufhören würden, wenn dieses Zweck aufhört; so ist der Trieb zu Erreichung ihres Zweckes so genau mit dem Erhaltungstriebe ihrer eignen Existenz verbunden, daß sie alles ausbietet müßen, um nicht mit dem Verluste ihres Zweckes selbst verlohren (!) zu seyn <um nicht in diesen Falle gewiß verlohren zu seyn $>$, da sie durch höchstmöglichen Beisteuer (§) dennoch ihre Existenz durch den möglichen Sinn der Erreichung des Zweckes vi[e]lleicht retten können. 
(continued)

\section{CHAPITRE VIII.}

$\mathrm{X}$.

En quoi consistent les devoirs du nouveau souverain, la société, envers lui-même? Ils consistent dans la contribution de tous les moyens de culture réunis aux moyens naturels de chacun de ses membres, afin d'arriver par elle à la satisfaction la plus entière de la faculté appétitive perfectionnée et développée, l'instinct formé pour le principe, moyens dont la réunion présente la propriété du nouveau souverain, c'est-à-dire le trésor social, et qui sont obligés de se prêter un secours mutuel relativement au but exigé par le principe, c'est-à-dire, pour pouvoir se conserver dans l'état de culture.

\section{Abtheilung VIII}

10.

Was sind die Pflichten des neuen Souverains (die Sozietät) gegen sich selbst?

Sie sind die Beisteuer aller zu ihren Naturmittels hinzu gekommen Kulturmitteln zur Erreichung ihres nun ausgedehnten Zweckes - der höchsten Befriedigung ihres neuen Leiters, des erhöhten und entwickelten Begehrungsvermögens, des ausgebildeten Instinktes (§ 4) - welche zusammengesetzten des Eigenthum (§ 6) des neuen Souverains (der Tresor Sozial) ausmachen, und sich untereinander reziprok beyhülfen müßen, um des Zweckes willen, von welchen ihre Erhaltung abzwingt. 